\title{
Angio CT assessment of anatomical variants in renal vasculature: its importance in the living donor
}

\author{
Julio Arévalo Pérez • Francisco Gragera Torres • \\ Alejandro Marín Toribio • Laura Koren Fernández • \\ Chawar Hayoun • Isaac Daimiel Naranjo
}

Received: 10 July 2012 /Revised: 22 November 2012 / Accepted: 19 December 2012 / Published online: 26 January 2013

(C) The Author(s) 2013. This article is published with open access at Springerlink.com

\begin{abstract}
Background Renal vasculature is known for having a broad spectrum of variants, which have been classically reported by anatomists.

Methods The distribution and morphology of these variations can be explained by considering the embryology of the renal vessels. With the recent outburst of imaging techniques, it has been the radiologist's turn to take the baton, recognising and describing unconventional renal vascular patterns.

Results Knowledge of these patterns has gained significance since the advent of the era of transplantation. For almost 60 years cadaveric donation has been the main source of kidneys suitable for transplantation. Living kidney donation demonstrates many advantages and stands out as the best alternative for organ procurement to meet the increasing demand. Since the dawn of laparoscopic nephrectomy as the technique of choice for organ procurement in living kidney donors, MDCT plays a key role as a noninvasive preoperative planning method for anatomic evaluation. As the field of view at laparoscopic surgery is limited, it is essential to meticulously assess the origin, number, division and course of arteries and veins.

Conclusion Awareness of the different anatomical variants allows the radiologist to enlighten the surgeon in order to avoid compromising the safety of the surgical procedure that could lead to significant complications.
\end{abstract}

J. Arévalo Pérez $(\triangle) \cdot$ F. Gragera Torres $\cdot$ A. Marín Toribio

L. Koren Fernández $\cdot$ C. Hayoun · I. Daimiel Naranjo

Department of Radiology, Hospital Universitario 12 de Octubre,

Avda de Córdoba s/n,

Madrid 28041, Spain

e-mail: arevaju@hotmail.com
Teaching Points

- Renal vasculature has many variants, which can be explained by considering the embryology of kidneys.

- Living kidney donation demonstrates many advantages over cadaveric donation.

- Angio CT evaluation of living kidney donors is a multiple phase study.

- A detailed report describing the variants, their distribution and morphology will help surgeons.

Keywords Living donor · Multidetector computed tomography $\cdot$ Embryology $\cdot$ Kidney transplantation .

Anatomy

\section{Introduction}

Renal vessels are acknowledged for presenting a wide range of variations. The study of the renal pedicle and its peculiarities has been of interest to anatomists since the end of the nineteenth century. The distribution and morphology of these anomalies can be explained by considering the embryological development of the renal vasculature, but it was after the outburst of imaging techniques that radiologists started recognising and describing unconventional renal vascular patterns, which led to a systematic clinical approach to this topic. Knowledge of these patterns has gained significance with the advent of the era of transplantation. Thanks to its success, this now everyday practice has enormously increased the need for organs. Given this growing demand, kidneys from living donors have been considered as an alternative to cadaveric donation to expand the pool of organs. Living kidney donation demonstrates many advantages and stands out as the best alternative for organ procurement. There has been a significant reduction of waiting 
time for transplantation since it can be done on a scheduled basis, even before entry into dialysis programmes [1]. When performed in this manner, an optimisation of conditions of donor and recipient, such as minimum ischaemia time, can be achieved. There is a lower incidence of delayed graft function, which is a negative prognostic factor for graft survival [1]. This fact allows not only for better graft endurance but also superior recipient survival compared to transplantation with a cadaveric donor. There is a reduced incidence of rejection and therefore less need for immunosuppressive drugs. As a consequence, not only more organs are available, but also there is less need for retransplantation. Furthermore, live donor transplantation is more cost effective than cadaveric transplantation [1].

It was due to a great disproportion between organ supply and demand that laparoscopic live donor nephrectomy was conceived as an effort to meet the ever-growing demand and thus reduce the shortage for organs $[1,2]$.

Laparoscopic living donor nephrectomy has become the technique of choice over laparotomy to increase organ availability. It has demonstrated a significant reduction of pain and morbidity, resulting in less use of analgesics and less frequent and generally milder postoperative complications, the most common being acute urinary retention and paresthesias. Both hospital stay and recovery time are reduced compared to laparotomised donors, permitting the patient to resume the activities of daily living and return to work sooner. Thanks to the better cosmetic results and a reduction of pain, it has been hypothesised that more people would be interested in donation. However, the involvement of an invasive intervention in a previously healthy candidate demands unique management and care. It is here where radiologists play an essential role in the anatomical evaluation of kidneys, placing special emphasis on the importance of certain relevant findings prior to nephrectomy $[1,2]$.

\section{Angio-CT}

\section{Technique}

Traditionally, living renal donors underwent radiographic evaluation with intravenous urography (IVU) and renal and aortic angiography. Depiction of renal size, calculi, calcifications and anatomy of the collecting system and arteries was fulfilled with these two techniques; however, renal masses, small lithiasis and venous anomalies were not properly assessed. For these and other reasons, the procedure has been replaced with $\mathrm{CT}$ evaluation since it offers unquestionable advantages [3]. CT angiography is less invasive, better tolerated by donors, and provides significantly more information than intravenous urography and angiography together, especially regarding abdominal anatomy and vascular pathways [4-6]. Previous works have shown that CT angiography has $100 \%$ sensitivity identifying accessory arteries and $93 \%$ sensitivity identifying prehiliar arterial branches [7]. The study consists of only one technique and can be done in just 1 day, as opposed to IVU and angiography, which needed a minimum of 2 days because of contrast restriction. This fact also implies an overall cost reduction of the procedure that has been reported to be of 35-50\% [8].

The most essential step in a CT angiography is undoubtedly the acquisition of raw data. The value and accuracy of the volumetric study will depend on this first stage [9-12]. Optimal timing of image acquisition and meticulous management of the patient, including preparation, positioning and contrast injection technique, is crucial. The technical parameters for the MDCT protocol depend on the speed of the scanner, the section thickness and the number of phases - in the majority of institutions the study comprises a multiple phase procedure including at least two of the following phases: unenhanced, arterial, nephrographic and delayed.

The main aim of the unenhanced phase is to locate the kidneys, rule out calculi and provide a baseline study to compare the enhancement of eventual lesions. The optimal timing for the contrast-enhanced phases depends on the volume of contrast material, the administration rate and the individual's cardiac output - these factors mandate a delay time between the start of the contrast introduction and the beginning of the scan [3]. An arterial phase can be programmed empirically $15-20 \mathrm{~s}$ after the injection of contrast material. However, using a bolus tracking detection system with the ROI placed in the abdominal aorta guarantees the success of the arterial phase acquisition. The scan covers from the top of the kidneys to the sciatic notch, and it allows the depiction of not only the renal arteries but also the renal veins. A nephrographic phase $65-90 \mathrm{~s}$ after the injection provides a proper enhancement of both renal and small tributary veins. For arterial and nephrographic phases, a minimum of 1-mm sections should be used for a better visualisation of the lumbar veins and accessory renal arteries because they can be small and easily missed when thicker sections are used [3]. Finally, a delayed phase $7-10 \mathrm{~min}$ after contrast injection is used to assess the renal collecting system and ureters.

Despite the benefits of using all four phases, there are consistent reasons to restrict this number: to limit the radiation dose to healthy subjects and to prevent from certain technical limitations like image noise and anode heating. To cope with these inconveniences, the authors propose different procedures: Kawamoto et al. dispense of the precontrast phase since, according to their experience, nephro and urolithiasis can be detected on arterial phase images with careful scrolling through continuous images or using maximum intensity projection (MIP). In addition, they also replaced 
the nephrographic phase with a venous phase with a 55 -s delay, which is not optimal for depiction of renal neoplasms, a criterion for exclusion as a donor [14]. Reducing the kilovolt peak $(<100 \mathrm{kV})$ in the precontrast phase results in more image noise, yet the images are still diagnostically satisfactory and the radiation dose is significantly reduced [13]. The delayed phase can be replaced by either a delayed topogram or conventional radiography to evaluate the excretory system, thus limiting the radiation dose (Table 1). A single topogram is not always sufficient to assess the collecting system, but in most cases it is. In fact, most published authors agree with the use of a delayed topogram with the goal of reducing radiation or even conventional radiography [3, 6-8]. Although it is assumed that taking the patient to the $\mathrm{x}$-ray facility would be more complicated and time consuming, a topogram in the modern CT scanners has a high-quality image. However, in case of doubt, an extra phase can be acquired to resolve uncertainties, always with a low-dose acquisition.

\section{Volumetric reconstruction techniques}

Once raw data are acquired, the next step is to generate axial images through a process called data or image reconstruction. Axial data can be directly analysed or used to produce multiplanar images. Multiplanar reformation (MPR) is a technique that processes information from axial CT images to create non-axial two-dimensional coronal, sagittal, oblique or curved plane images. MPR images can, in turn, be thickened into slabs by means of projectional techniques [19], thus displaying images that can emulate angiograms.

Table 1 Angio-CT protocol for assesment of potential living renal donors

\begin{tabular}{ll}
\hline Parameter & Protocol \\
\hline CT scanner & Philips Brilliance 16 \\
Voltage & $120 \mathrm{kVp}$ \\
Effective current & $144-210 \mathrm{~mA}$ \\
Rotation time & $0.5 \mathrm{~s}$ \\
Detector collimation & $16 \times 0.75$ \\
Slice thickness & $1 \mathrm{~mm}$ \\
Slice increment & $0.5 \mathrm{~mm}$ \\
Intravenous contrast material & $2 \mathrm{ml} \times \mathrm{kg}$ body wt $+40 \mathrm{ml}$ saline \\
Injection rate & $4 \mathrm{ml} / \mathrm{s}$ \\
Basal precontrast & $<100 \mathrm{kV}$ \\
Arterial phase & Basal, arterial (bolus tracking at \\
& $150 \mathrm{HU}$ with $10-\mathrm{s}$ delay) \\
Nephrographic phase & $65-\mathrm{s} \mathrm{delay}$ \\
Delayed topogram & 5 -min delay \\
Reconstruction & $50 \%$ overlap \\
\hline
\end{tabular}

All phases include a dose modulation protocol
There are two main techniques used to perform CT angiography.

MIP (maximum intensity projection) is a reconstruction algorithm that selects and displays only the voxels with highest attenuation value of a selected slab in the visualisation plane [19]. It is best used to evaluate the brightest objects in the image, such as contrast materialfilled structures [19, 21]. This is why MIP has been proven to be precise for the evaluation of the vasculature and thus is widely employed in CT angiography. Nevertheless, for a correct interpretation of MIP images, it is necessary to be familiar with the limitations of this algorithm: When evaluating atherosclerotic vessels, the presence of high attenuation voxels, such as calcifications, limits the assessment of the vascular lumen by overestimating stenosis [20, 22-24]. Since it is a twodimensional representation and it does not provide a good sense of depth, MIP is not able to demonstrate the 3D relationships of the vessels [19, 20, 23, 25]. To include long segments of vessels in CT angiography, thick-slab MIP images can be helpful, but small arteries should be evaluated with thin sections viewed in sequence because usually they are not visible because of their low attenuation [19]. Evaluation of vessels can be improved by eliminating high-density structures such as bone through slab editing [19, 26, 27].

Volume rendering is an effective modality for the 3D display of imaging data. It assigns opacity values on a full spectrum from $0 \%$ (transparency) to $100 \%$ (opacity) along an artificial line of sight projection [19, 28, 29]. By combining opacity values and lighting effects, volume rendering allows for an appreciation of spatial relationships. It also provides a good differentiation of two structures of little difference in HU that are close in space to each other, permitting a better visualisation of vessels compared to MIP, and since bones do not obscure the visualisation of vessels, therefore there is no need to perform slab editing [20]. Also, volume rendering permits a better visualisation of soft tissue and 3D relationships.

Radiologists should be aware of advantages and disadvantages of these techniques and how they complement each other: MIP excels at displaying vascular maps and small intraparenchymal vessels in enhancing organs (Fig. 1), whereas volume rendering is better at depicting small extraparenchymal vasculature, grading intraluminal stenosis with calcium and demonstrating 3D relationships between structures [20, 30, 31] (Fig. 2).

\section{MRA vs. CTA}

Some authors have proposed that MRA could replace MDCT as a preoperative assessment method on renal donors, avoiding exposure to radiation and nephropathy 


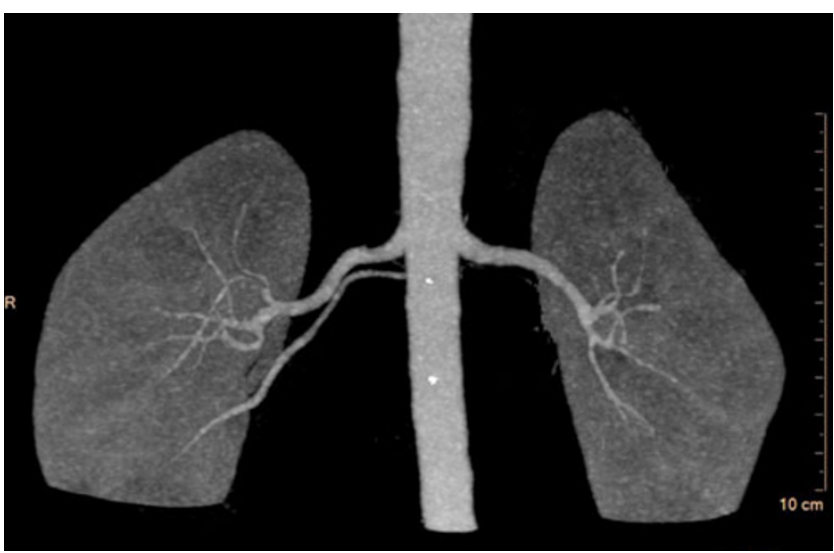

Fig. 1 MIP image shows an accessory right renal artery arising from the anterior aspect of the abdominal aorta. Note the better visualisation of intraparenchymal vessels as well as the lack of sense of depth of the structures displayed

due to contrast agents [15]. While the reduced radiation exposure is a benefit, most donors are healthy individuals with a normal renal function that rarely develop contrastinduced nephropathy. Moreover, there are some limitations regarding MR angiography: Motion artefacts are more frequent in MRA studies since patients have to hold their breath longer, and the study also requires more time to complete. Angio CT permits higher resolution, given that MRA pulse sequences do not allow scanning with a thickness of $1 \mathrm{~mm}$ or less. There are some artefacts that are more likely to occur in MRA and can compromise image quality, such as phase encoding artefacts, vascular pulsation and chemical shift artefacts at fat-soft tissue interfaces, especially in the retroperitoneum. All these artefacts can cause misdiagnosis of small vessels. In addition, the cost of MRA makes it a less accessible technique in most centres [16-18].

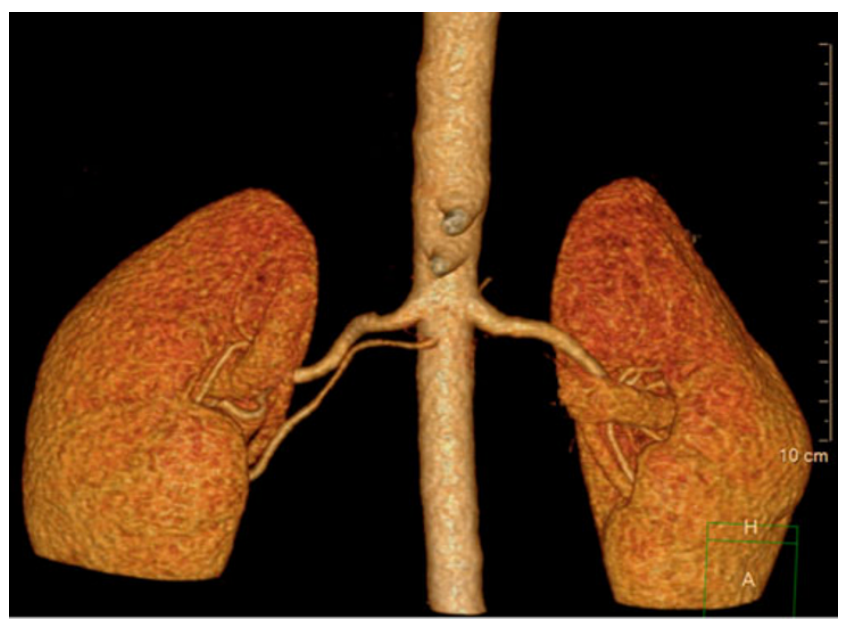

Fig. 2 Volume-rendered image of the same structures offering an improved display of both extraparenchymal vessels and 3D relations between them

\section{Renal arteries}

Embryology

The variations in the distribution and morphology of renal artery supply can be explained by considering the embryology of the renal vasculature. The most widely accepted and frequently quoted theory was proposed by W. Felix and provides a convenient embryological explanation of the diversity of renal, adrenal and gonadal arteries. The mesonephros, in a 5 th week human embryo, is irrigated by a group or arteries arising from the lateral surface of the abdominal aorta (Fig. 3a). Initially they are distributed within the mesonephric area, but later they also supply the reproductive glands, suprarenal bodies, metanephros and diaphragm. The number of mesonephric arteries is not constant, and it follows the degeneration of the mesonephros with the progressive craniocaudal development of the embryo. As growth progresses, arteries recede into the lumbar segments where mesonephric tubules are more abundant, thus disappearing from the thoracic segments. From the 10th thoracic to the 3rd lumbar segment, all the mesonephric arteries may persist. Considering these persisting arteries in detail, they can be divided into three groups in the region between the superior and inferior mesenteric arteries: a cranial, middle and caudal group. The latter originates a network, the rete arteriosum urogenitale, which consists of approximately 20 segmental branches that supply the mesonephros, reproductive gland and metanephros. Should some of these arteries degenerate, adjacent vessels take their place. The metanephros or 'future kidney' climbs upwards from the pelvic to the lumbar region during the earlier morphogenetic period. After achieving enough cranial blood supply, caudal arteries regress. By the time it reaches its final position in the lumbar region, it is supplied by various arteries. One of these becomes the main renal artery while the rest either disappear or persist as accessory renal arteries (Fig. 3b). The definitive renal artery develops either from the last branch of the middle group or the first of the caudal group. The relations between the rete urogenitale and the metanephros illustrate the development of accessory arteries and their relation to the kidney. The suprarenal arteries mostly arise from the middle group, but can also form from the cranial group just like the inferior phrenic. Mesonephric arteries supplying the reproductive gland form the gonad arteries, hence the varied number and origin of these arteries. The irregular development of this model explains why persisting vessels form the definite segmental arteries of the adult kidney, having variations at their point of origin [32].

\section{Arterial anatomy}

In most individuals, the kidneys are supplied by only a pair of arteries arising from the abdominal aorta below the 
a

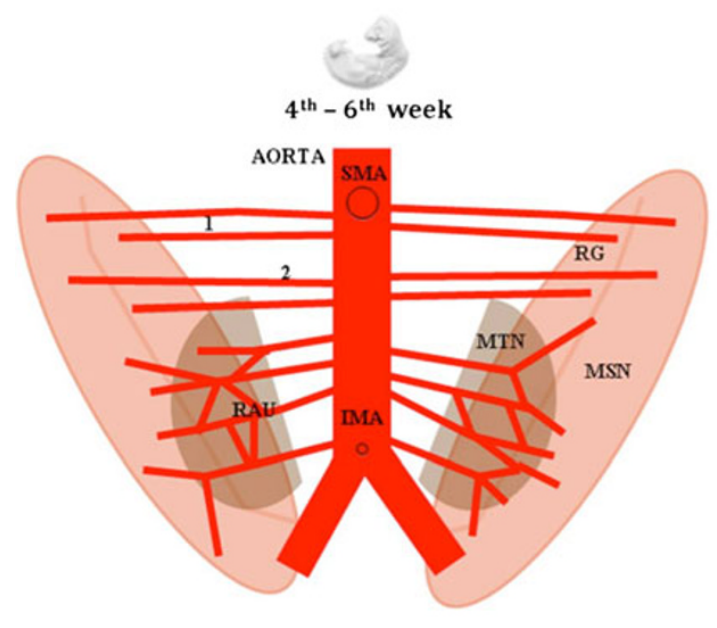

b

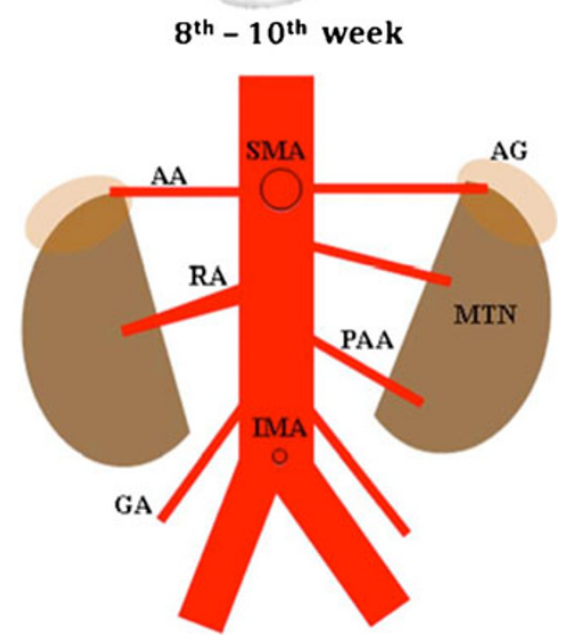

Fig. 3 Diagrams showing arterial mesonephric blood supply and its relationship to eventual metanephros in a 4-6-week embryo and 8-10week embryo. SMA: Superior mesenteric artery, IMA: inferior mesenteric artery, 1: cranial group mesonephric arteries, 2: middle group mesonephric arteries, RG: reproductive gland, MSN: mesonephros, MTN: metanephros, RAU: rete arteriosum urogenitale, SMA: superior mesenteric artery, IMA: inferior mesenteric artery, GA: gonadal artery, AG: adrenal gland, AA: adrenal artery, MTN: metanephros, RA: renal

origin of the mesenteric artery at the level of the L1 to L2 vertebral bodies. Each renal artery is divided into anterior and posterior branches (presegmental arteries) at the hilium of the kidney. It further divides into segmental arteries to supply the respective segments of the kidney (apical, upper, middle, inferior and posterior). It has been demonstrated that there is a segmental arrangement of these vessels that is stable, and there is no collateral arterial circulation between the segments, establishing that each of the segments is supplied by their own segmental artery [33]. But this segmental model is not constant, and variations in renal arteries, including the number, source and course, are very common. They are often related to malpositioned or malrotated kidneys, but have also been described in up to a $30 \%$ of cases in dissection series in orthotopic kidneys [7, 8, 34-36] (Figs. 4 and 5). These variations are probably the result of an uneven degeneration of the rete arteriosum urogenitale and the rest of the mesonephric arteries. This may explain why there is some variation in the point of origin of those arteries that persist to form the final segmental arteries of the adult kidney.

\section{Arterial variants}

The diversity in renal arterial anatomy can be classified according to the number, division pattern as well as distribution/origin of the arteries. Accessory renal arteries can arise from the abdominal aorta above the main branch or artery, PAA: polar accessory artery. The adrenal artery usually develops from the cranial group of mesonephric arteries whereas the gonadal artery forms from arteries of the caudal group (RAU). The definite renal artery comes either from the last branch of the middle group or the first of the caudal group. Persisting mesonephric arteries form accessory vessels (PAA) as shown in the example

as low (inferiorly) as the internal iliac artery. Varied origins such as the iliac, superior and inferior mesenteric, celiac, middle colic, lumbar, middle sacral and contralateral renal arteries have been reported [35, 37] (Fig. 6).

Considering the division pattern, a common variant is when the main renal artery divides before it reaches the renal hilum. This condition is named the extrahiliar branching $[7,8,35]$ (Fig. 7). Should it take place within $1.5 \mathrm{~cm}$ of the renal ostium in the abdominal aorta, it is called early branching [7] (Fig. 8). It is essential to identify any prehilar

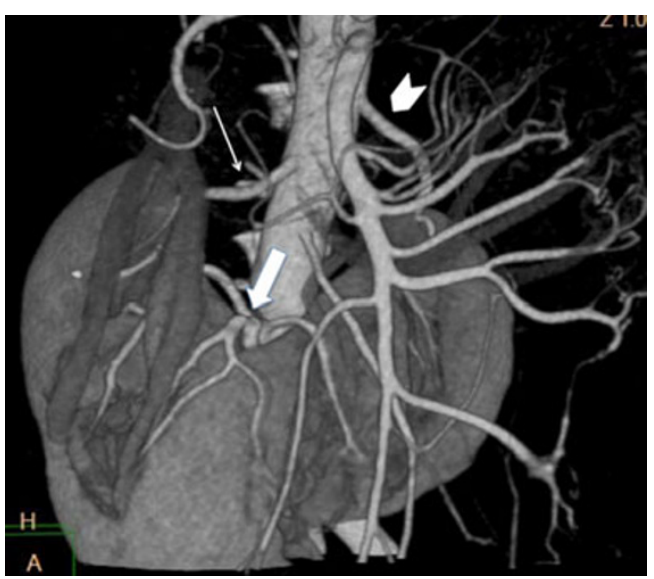

Fig. 4 Volume-rendered image of a horseshoe kidney demonstrating a triple arterial system: a right renal artery (thin white arrow), a central inferior renal artery that supplies the isthmus (thick white arrow) and a left renal artery (arrow head) 


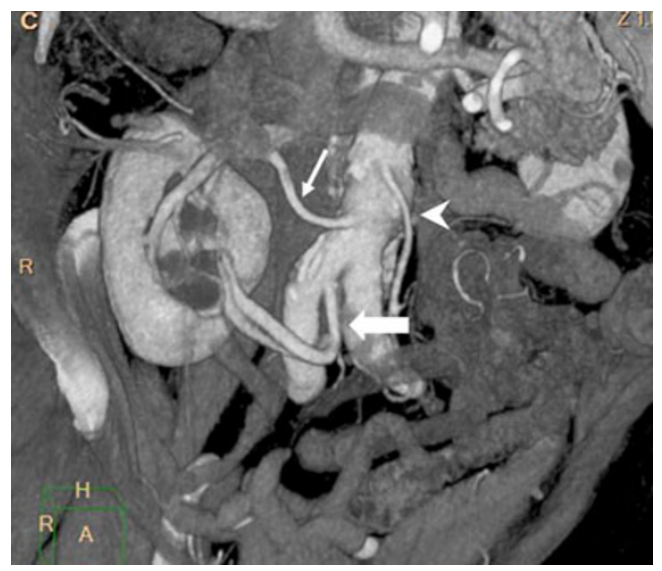

Fig. 5 Volume-rendered image of a pelvic ectopic right kidney, which has two renal arteries, one of which originates in the anterior plane of the aorta (thin white arrow), below the origin of the inferior mesenteric artery (arrow head), and the other arises from the medial aspect of the right common iliac artery (thick white arrow)

branching that occurs within $2 \mathrm{~cm}$ of the origin of the renal artery from the abdominal aorta, since most surgeons require at least a 2-cm length of renal artery before hilar branching to guarantee satisfactory control and anastomosis [14]. According to Raman et al., early branching of the left renal arteries $(<2 \mathrm{~cm}$ from the aorta) is present in $21 \%$ of cases whereas early branching of the right renal arteries is present in $15 \%$ of individuals [38].

When there are two or more arteries with a separate origin, the vessel with the greatest diameter is considered as the main renal artery, and the rest are branded as accessory or supernumerary. Additional arteries can, in turn, be divided into two categories according to how they attain the kidney: hiliar, entering at the hilium, and polar, reaching at the pole [39]. Multiple renal arteries can be seen in one-third of patients. In a study with 800 kidneys from cadaver donors, Pollak et al. demonstrated $23 \%$ double arteries,

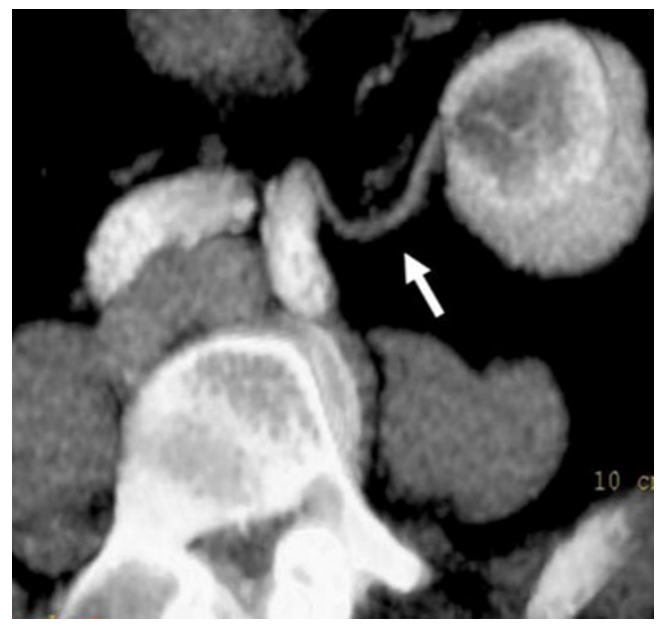

Fig. 6 MIP image showing a polar accessory renal artery (white arrow) arising from the left common iliac artery supplying the inferior renal pole

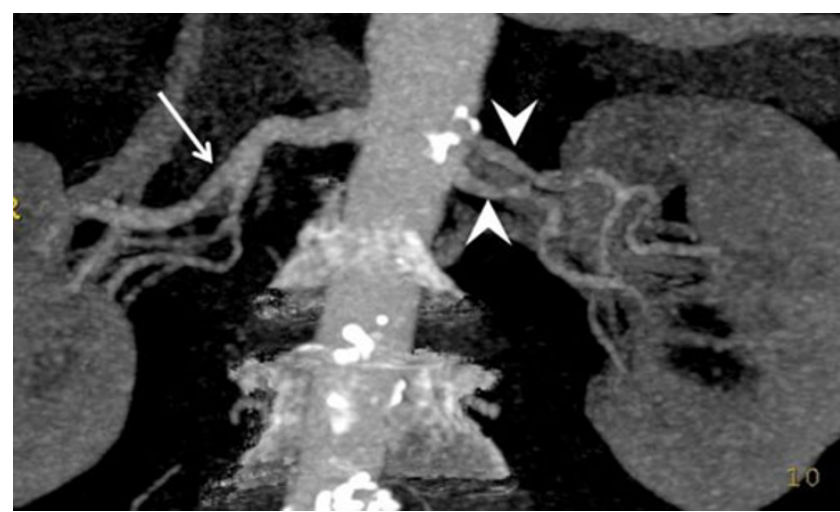

Fig. 7 MIP image presenting right extrahiliar branching (thin white arrow) as well as a double left renal artery arising from the abdominal aorta (arrowheads)

$4 \%$ triple arteries and $1 \%$ quadruple arteries [40]. This multiplicity is more frequently unilateral than bilateral [23]. The most frequent accessory artery is a polar artery arising from the aorta, close to the origin of the main renal branch, and supplies the inferior renal pole (Fig. 9). The second most frequent supplementary artery irrigates the upper pole, which is normally a small segment [40]. Some authors call polar arteries those that attain any of the kidney poles with independence of its origin. It may be helpful, strictly from a labelling point of view, to distinguish between these polar arteries, tagging them as polar branches when they arise from the main renal artery and polar accessory arteries when they have a separate origin. The importance of polar arteries is shown in the fact that they supply renal parenchyma, and when damaged during nephrectomy it can cause arterial bleeding or renal infarction. A case particularly worth mentioning is inferior polar arteries that provide vessels for the upper excretory system. A section of an inferior polar artery can cause pyeloureteral necrosis of the graft leading to stenosis or urinary tract leakage. If it is not possible to

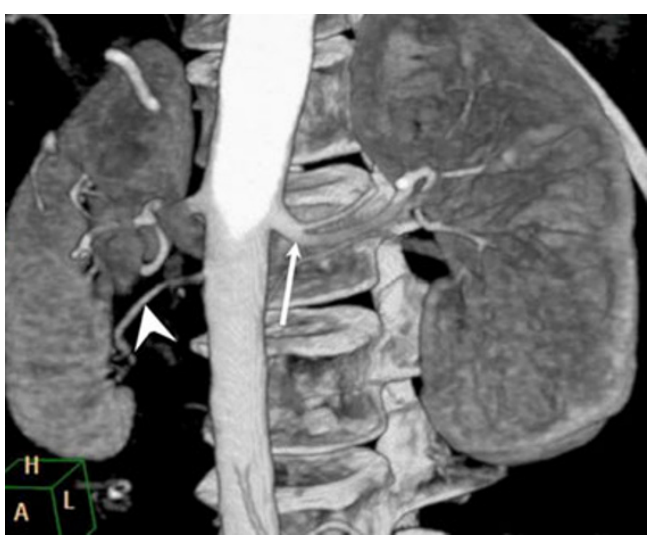

Fig. 8 Volume-rendered image illustrating early branching of the left renal artery (white arrow) where the renal artery branches within $1.5 \mathrm{~cm}$ from the ostium. The right kidney presents an inferior polar accessory artery (arrowhead) 


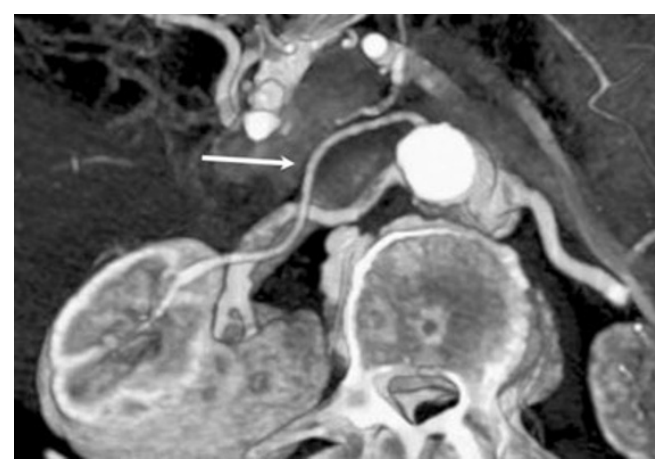

Fig. 9 Volume-rendered image where a right renal accesory artery (white arrow) arises from the abdominal aorta following a precaval trajectory to irrigate the inferior pole of the right kidney

preserve the artery, a pyeloureteral anastomosis between the graft and the recipient can be considered [41] (Figs. 10a, b, 11 , and 12).

\section{Renal veins}

\section{Embryology}

The embryo's venous system is composed of a very irregular network of capillaries from which individual ones transform definitively into veins while others disappear. As a result the venous system is not very uniform, and in the adult we can find far more variants of veins than on the arterial side. We will focus on the development of the renal veins and their primitive origin, the subcardinal veins and their anastomoses that explain the multiplicity of variants that we can find.

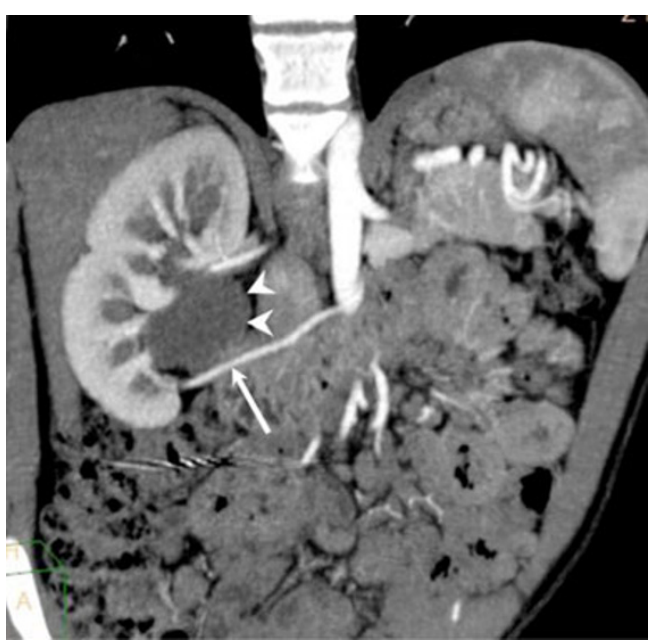

Fig. 11 Average coronal reconstruction demonstrating the polar accessory artery (white arrow) producing a compression of the pyeloureteral junction and significant pyelocalyceal dilatation (arrowheads)

Throughout the 4th week the posterior or inferior cardinal veins, which drain the inferior part of the embryo, are formed. The mesonephros drains in the subcardinal veins that develop during the 5th week. They are located ventromedially to the mesonephros and run parallel to the posterior cardinal veins. Subcardinal veins connect with the posterior cardinal veins through multiple anastomoses (intersubcardinal anastomoses). Persistence of some of these anastomoses explains many of the variants we can find. During the 6th week, the supracardinal veins develop lying medially to the posterior cardinal veins, assuming the drainage of the body wall after the obliteration of the major portion of the posterior cardinal veins (Fig. 13a). As the mesonephros regresses, the metanephros and adrenal gland expand and ascend, while the gonads descend [32]. This circumstance forces
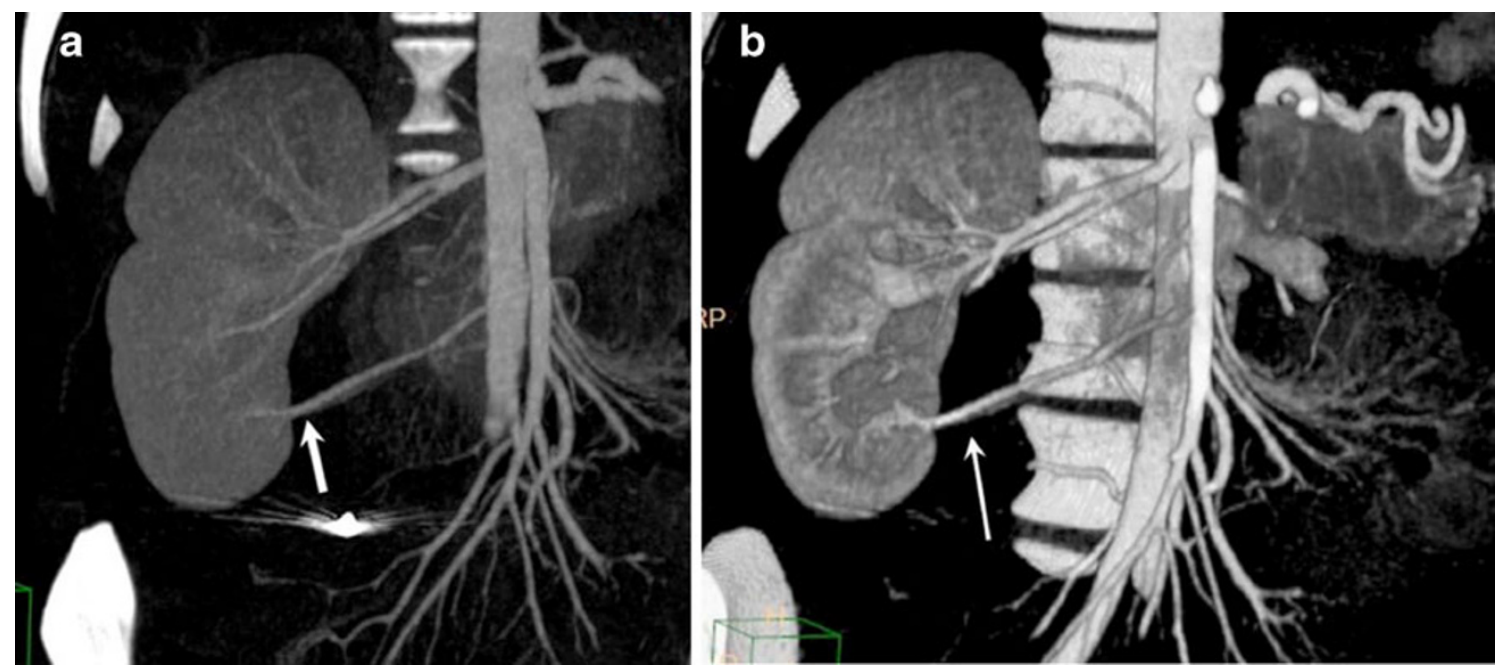

Fig. 10 a MIP image illustrating a right inferior polar accessory artery (white arrow) arising from the anterior aspect of the aorta. b Volume rendered image illustrating a right inferior polar accessory artery (white arrow) arising from the anterior aspect of the aorta. 


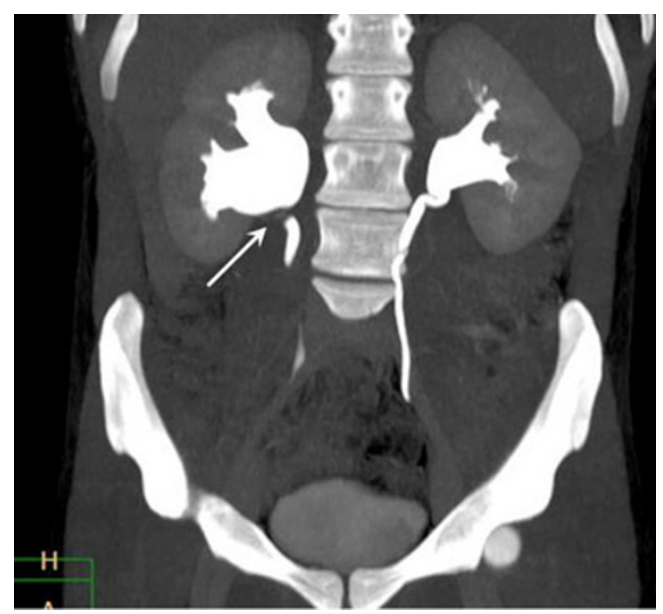

Fig. 12 Coronal MIP reconstruction of the excretory phase demonstrating the stenosis (white arrow) in the pyeloureteral junction

drastic changes not only in their respective arterial systems, but also in their venous drainage organisation, which adopts more medial drainage routes to the anastomotic channels between the supracardinal and subcardinal veins. These anastomotic channels between the supracardinal and subcardinal veins transform into the renal segment of the inferior vena cava (IVC) [42]. After this communication has been established, the left subcardinal vein disappears, remaining only its distal portion as the left gonadal vein. Hence, the right subcardinal vein is transformed into the main drainage route, forming the suprarenal segment of the IVC, whereas the supracardinal veins contribute to the development of the infrarenal part of the IVC and to the azygos and hemiazygos vein systems (Fig. 13b) [42].

\section{Venous anatomy}

Following the venous flow, the renal cortex is drained consecutively by the stellate veins, the arcuate and interlobar veins [23, 43-46]. It has been demonstrated that, as opposed to the intrarenal arteries, the intrarenal veins show no segmental arrangement at all, there being a free anastomosis of the venous system throughout the kidney. These anastomoses occur at different levels between the stellate veins (cortex), between the arquate veins (base of the pyramids) and between the interlobar veins (close to the renal sinus). There are also anastomoses around the calyceal necks that join a longitudinal system that ends up forming big trunks that finally form the main renal vein [47]. Thanks to this network, ligation of the venous branches can be performed if one vessel is cut or damaged during surgery, permitting an alternate flow and avoiding the risk of parenchymal loss. The renal vein is usually located anterior to the a

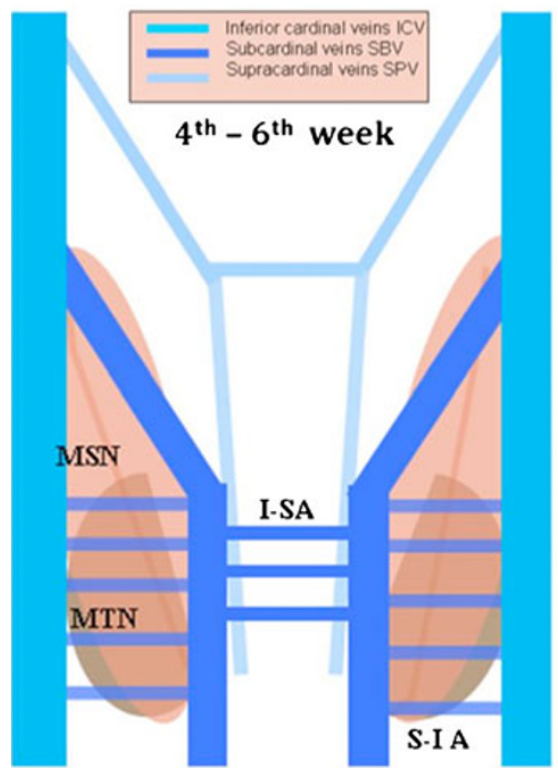

Fig. 13 Diagram showing the development of the embryo's inferior venous system in a 4-6-week embryo and 8-10-week embryo. I-SA: Intersubcardinal anastomoses, S-IA: subcardinal-inferior cardinal anastomoses, MSN: mesonephros, MTN: metanephros, AZG: azygos vein, IV: iliac veins, HZG: hemiazygos vein, AG: adrenal gland, GV: gonadal vein, LV: liver. There are three main venous systems that contribute to the formation of the renal veins and the inferior vena cava. The right subcardinal vein constitutes the suprarenal segment while the b

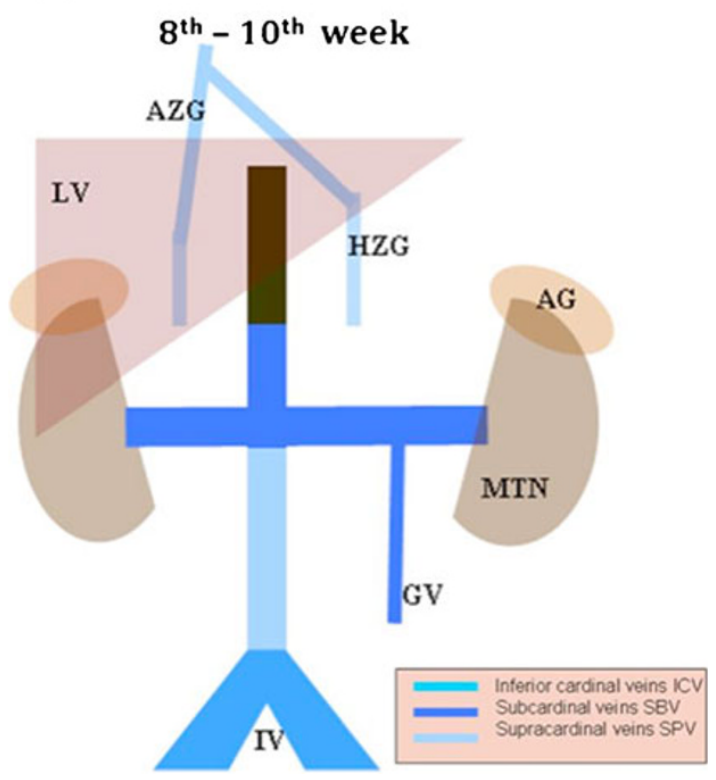

I-SA derives into the left renal vein. The hepatic segment forms from the vitelline vein. The azygos and hemiazygos systems are formed from the cranial part of the SPV. The caudal part of the right SPV persists as the infrarenal segment of the IVC. The left subcardinal vein regresses partially, remaining its distal portion as the left gonadal vein. Finally, the persisting segment of the inferior/posterior cardinal veins forms the iliac veins 
Fig. 14 Volume-rendered image showing multiplicity of the right renal vein (white arrows). Note the different drainage first to the IVC (a) and then to the main renal vein $(\mathbf{b})$
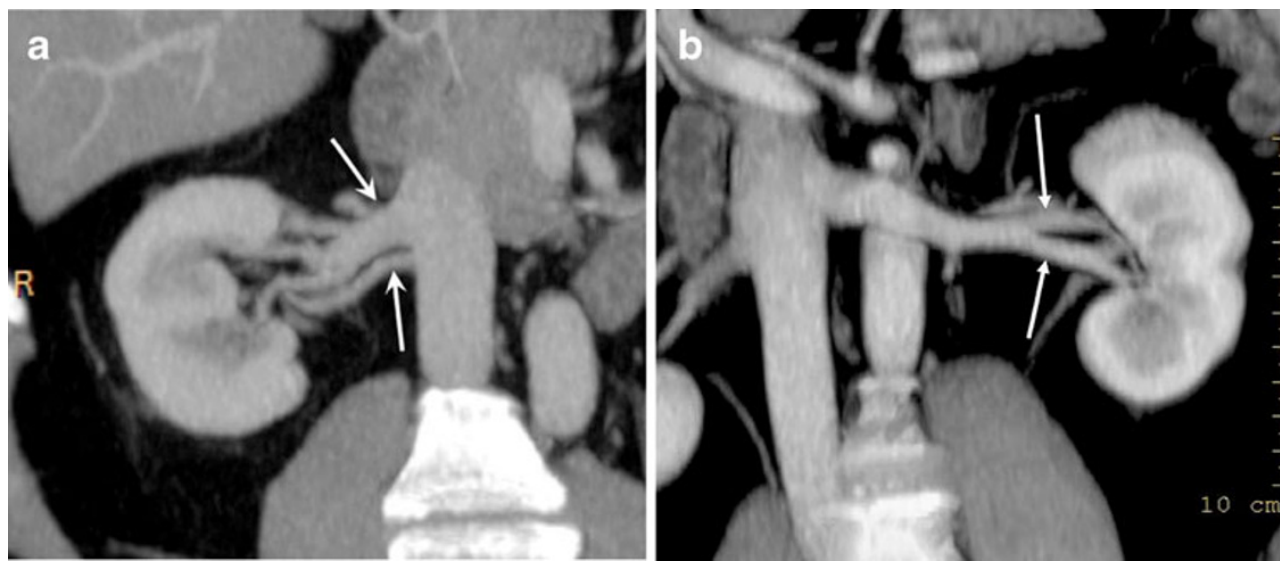

renal artery at the renal hilum [23]. Around $85 \%$ of the population have just one right renal vein. It is about 2$2.5 \mathrm{~cm}$ in length and follows an anterosuperior trajectory, reaching the IVC at the level of the inferior margin of L1 [7]. Normally no other extrarrenal veins attain the right renal vein. The left renal vein is on average $8.5 \mathrm{~cm}$ in length. It normally takes a transverse and ventral course between the superior mesenteric artery and the abdominal aorta before reaching the inferior vena cava at the level of L2-L3. As opposed to the right renal vein, the left renal vein receives several tributaries before joining the inferior vena cava $[7,37]$. It receives the left adrenal vein superiorly and that in turn receives the inferior phrenic and capsural veins. The left gonadal vein joins the left renal vein inferiorly. The posterior aspect is attained in $75 \%$ of cases by retroperitoneal veins (lumbar, ascending lumbar and hemiazygos) [7].

Venous variants

The venous anatomy is not without variations - in fact, we can find far more variants of veins than on the

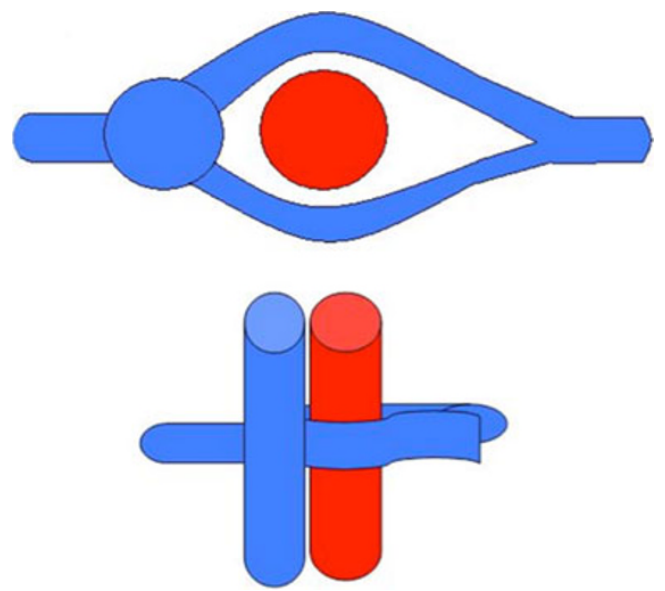

Fig. 15 Diagram illustrating disposition of a circumaortic left renal vein with both anterior and posterior branches surrounding the abdominal aorta arterial side. The most common is the existence of multiple renal veins, which occurs in $15 \%-30 \%$ of patients $[23,44]$. There are multiple right renal veins in $30 \%$ of cases, which consist of between two and four vessels that originate in the hilum [7, 48, 49] (Fig. 14a, b). A unique right renal vein, in $4 \%$ of individuals, may branch prior to its union with the IVC to let the right gonadal artery pass through. In $6 \%$ of right gonadal veins and $3 \%$ of retroperitoneal veins, they directly join the right renal vein instead rather than the IVC [7].

The most characteristic variant of the left renal vein is the circumaortic renal vein (17\% of patients), so named because it embraces the ventral and dorsal aorta [23] (Fig. 15). The circumaortic renal vein has two varieties. The most common is a single vein that originates in the hilum and splits right before surrounding the aorta (75\%), while in the less common variety the vein duplicates from its origin in the hilum [50] (Figs. 16 and 17). Typically, in the circumaortic variety the gonadal vein joins the dorsal branch and the adrenal vein the ventral branch [44]. In the embryo the left renal vein

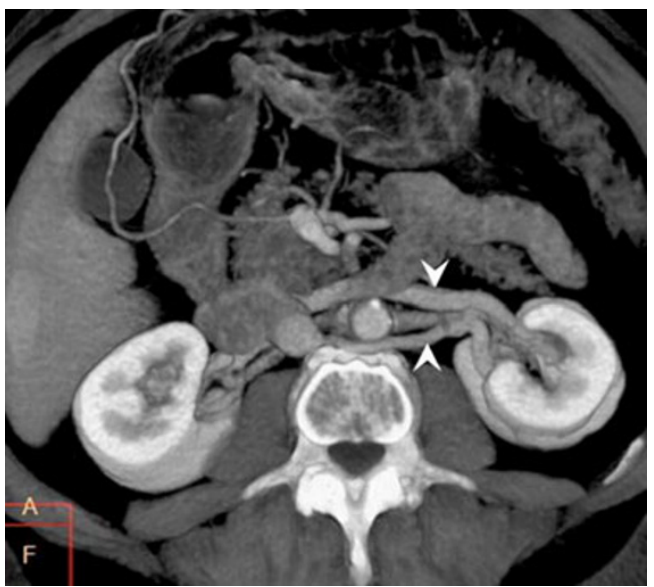

Fig. 16 Volume-rendered axial image demonstrating a circumaortic left renal vein with anterior and posterior branches (arrowheads) that embrace the abdominal aorta and converge in the hilum separately (the less common variant) 


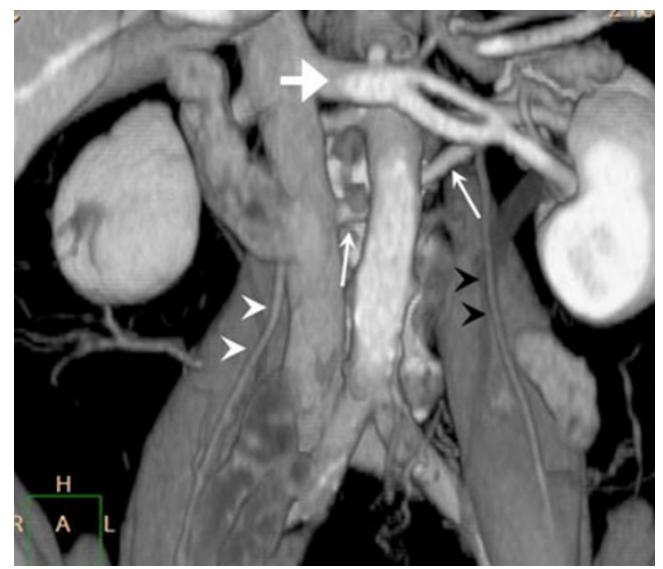

Fig. 17 Volume-rendered coronal image displaying a circumaortic renal vein. Note how the anterior branch (white thick arrow) forms a ring. The left gonadal vein (black arrowheads) joins the anteroinferior aspect of the ring. The posterior branch (white thin arrows) surrounds the posterior aspect of the aorta to finally reach the IVC. The right gonadal vein (white arrowheads) joins the IVC directly

develops from the intersubcardinal anastomoses, which course anterior to the aorta. Persistence of both the intersupracardinal and intersubcardinal veins forms the circumaortic ring, with one vein coursing anterior and the other posterior to the aorta [42].

The retroaortic renal vein is a less common entity. It has been reported in $3 \%$ of the population. It usually joins the lower lumbar portion of the inferior vena cava, though less frequently it can also drain into the iliac vein [51] (Figs. 18 and 19).

The left renal vein develops from the intersubcardinal anastomoses, which course anterior to the aorta. The retroaortic left renal vein occurs when the vein derives from the intersupracardinal veins, which are located posterior to the aorta $[42,52]$.

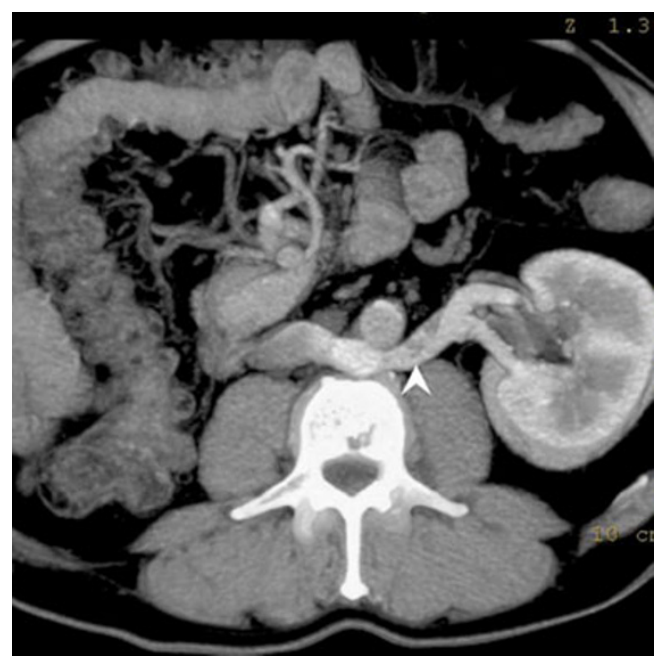

Fig. 18 Volume-rendered axial image showing a retroaortic left renal vein (arrowhead)

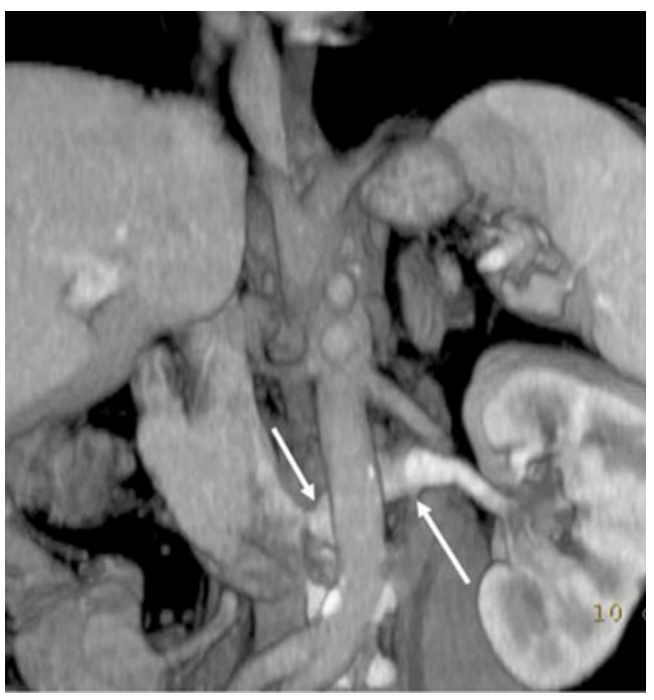

Fig. 19 Volume-rendered coronal image showing a retroaortic left renal vein (white arrow). Normally it joins the lumbar segment of the IVC although on rare occasions it reaches the iliac vein

\section{Other vessels}

Lumbar veins

Retroperitoneal veins (lumbar, ascending lumbar and hemiazigos) join the left renal vein in up to $75 \%$ of individuals, whereas in only a $3 \%$ they empty into the right renal vein [49] (Fig. 20). Normally left lumbar veins course ventrally and reach the posterior aspect of the left renal vein. Retroperitoneal veins draining into the left renal vein are of great importance, since they are variably sized and are often hidden during laparoscopy surgery. Clinically significant lumbar veins thicker

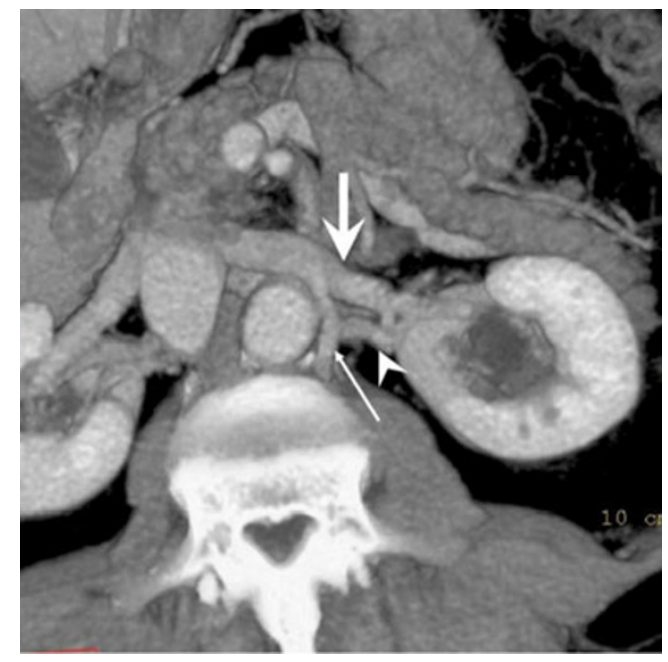

Fig. 20 Volume-rendered image displaying a posterior left renal vein (white arrowhead) draining in a lumbar vein (white thin arrow) that, in turn, joins the anterior left renal vein (thick arrow) 
than $3 \mathrm{~mm}$ can be identified using MDCT with high sensitivity and specificity.

MDCT does not consistently demonstrate veins smaller than $3 \mathrm{~mm}$, but these vessels are irrelevant from a clinical point of view as they could be cut and sealed during dissection without substantial bleeding [18]. With the goal of decreasing radiation, some authors have replaced multiphase scans by a single late arterial phase where renal veins can be identified. However, since retroperitoneal veins enhance more slowly, they should be properly evaluated in the venous phase, avoiding the risk of misdiagnosis that could compromise surgery. Leventhal et al. reviewed the result after 500 donor laparoscopic nephrectomies and reported 6 open conversions due to bleeding. Two out of the six (33\%) were due to lumbar vein injuries. They acknowledged that venous dissection represented the "most challenging aspect of the laparoscopic donor procedure", especially if lumbar branches were present $[18,53]$. Given the limitations in the field of view during laparoscopy, an accurate preoperative assesment of renal vessels, including the retroperitoneal veins, is crucial to prevent complications such as haemorrhage and gas embolism [18].

\section{Adrenal vessels}

Adrenal glands are irrigated by three different sources: the inferior phrenic arteries, renal arteries and aorta. They are supplied by all three in $34 \%$ of cases, by two in $61 \%$ and by a single vessel in $5 \%$. The main vascular contribution comes from the renal artery in $71 \%$ of cases.

A single right adrenal vein joins the IVC in most cases $(69 \%)$ while in $31 \%$ of individuals an accesory adrenal vein drains in the right renal vein. On the left side, almost constantly, the left adrenal vein joins both the left inferior phrenic and capsular veins to drain in the superior aspect of the left renal vein [7, 54].

\section{Gonadal vessels}

Gonadal arteries are paired vessels arising from the abdominal aorta at the level of L2, passing obliquely in a downward trajectory on the psoas muscle, and reaching the inguinal canal or the round ligament. In most individuals $(83 \%)$ they arise adjacent to each other from the anterior aspect of the aorta, below the origin of the renal arteries [7]. Arched gonadal arteries, arteries coursing anterior to both renal veins with an aortic origin, have been described in $20 \%$ of these cases [55]. They can also originate from the middle suprarenal artery and lumbar arteries. They may come from a common trunk and head into two or three branches. The most frequent variants are: a right gonadal artery arising from the right renal artery and a left artery with an aortic origin $(6 \%)$; a left gonadal artery starting at the level of the left renal artery with an aortic origin of the right gonadal $(4 \%)$; and gonadal arteries arising from the renal arteries (4\%) [7, 35].

In $93 \%$ of individuals the right gonadal vein reaches the IVC below the level of the renal veins, while in $7 \%$ of cases it joins the right renal vein. The left gonadal vein attains the left renal vein in most cases. Gonadal veins are $2-3 \mathrm{~mm}$ in diameter on average, growing up to $10 \mathrm{~mm}$ (mostly in post partum women). A gonadal vein with a significant diameter should be reported because the surgeon may need to use a different ligation procedure instead of cauterisation [39, 41].

\section{Conclusion}

ANGIO-CT plays a key role as a noninvasive preoperative method for assessment of possible kidney donors. Since renal vasculature presents a broad spectrum of variants, the goal of this evaluation is to determine if a patient is a suitable candidate for donor nephrectomy on the basis of the renal vascular anatomy. Awareness of the different variants together with a detailed report of their distribution and morphology also allows the radiologist to enlighten the surgeon in order to avoid compromising the safety of the surgical procedure, which could lead to significant complications.

Acknowledgments We would like to thank Alan Windhausen for his help with editing and language review.

Open Access This article is distributed under the terms of the Creative Commons Attribution License which permits any use, distribution, and reproduction in any medium, provided the original author(s) and the source are credited.

\section{References}

1. Ratner LE, Montgomery RA, Kavoussi LR (1999) Laparoscopic live donor nephrectomy: the four year Johns Hopkins University experience. Nephrol Dial Transplant 14:2090-2093

2. Ratner LE, Ciseck LJ, Moore RG, Cigarroa FG, Kaufman HS, Kavoussi LR (1995) Laparoscopic live donor nephrectomy. Transplantation 60:1047-1049

3. Rydberg J, Kopecky KK, Tann M et al (2001) Evaluation of prospective living renal donors for laparoscopic nephrectomy with multisection CT: the marriage of minimally invasive imaging with minimally invasive surgery. RadioGraphics 21: S223-S236

4. Alfrey EJ, Rubin GD, Kuo PC et al (1995) The use of spiral computed tomography in the evaluation of living donors for kidney transplantation. Transplantation 59:643-645

5. Lindgren BW, Demos T, Marsan R et al (1996) Renal computed tomography with 3-dimensional angiography and simultaneous measurement of plasma contrast clearance reduce the invasiveness and cost of evaluating living renal donor candidates. Transplantation 61:219-223 
6. Platt JF, Ellis JH, Korobkin M, Reige KA, Konak JW, Leichman AB (1996) Potential renal donors: comparison of convencional imaging with helical CT. Radiology 198:419-423

7. Pozniak MA, Balison DJ, Lee FT Jr, Tambeaux RH, Uehling DT, Moon TD (1998) CT angiography of potential renal transplant donors. RadioGraphics 18(3):565-587

8. Cochran ST, Krasny RM, Danovitch GM et al (1997) Helical CT angiography examination of living renal donors. AJR 168:15691573

9. Kuszyk BS, Heath DG, Ney DR et al (1995) CT angiography with volume rendering: imaging findings. AJR Am J Roentgenol 165:445-448

10. Smith PA, Fishman EK (1998) CT angiography: renal applications. In: Ferris EJ, Waltman AC, Fishman EK, Polak JF, Potchen EJ (eds) Syllabus: a categorical course in diagnostic radiology-vascular imaging. Radiological Society of North America, Oak Brook, pp 35-45

11. Kuszyk BS, Fishman EK (1998) Technical aspects of CT angiography. Semin Ultrasound CT MR 19:383-393

12. Smith PA, Fishman EK (1998) Three-dimensional CT angiography: renal applications. Semin Ultrasound CT MR 19:413424

13. Sahani DV, Kalva SP, Hahn PF, Saini S (2007) 16-MDCT angiography in living kidney donors at various tube potentials: impact on image quality and radiation dose. AJR Am J Roentgenol 188 (1): $115-120$

14. Kawamoto S, Montgomery RA, Lawler LP, Horton KM, Fishman EK (2004) Multi-detector row CT evaluation of living renal donors prior to laparoscopic nephrectomy. Radiographics $24: 453-466$

15. Willmann JK, Wildermuth S, Pfammatter T, Roos JE, Seifert B, Hilfiker PR et al (2003) Aortoiliac and renal arteries: prospective intraindividual comparison of contrast-enhanced threedimensional MR angiography and multi-detector row CT angiography. Radiology 226:798

16. Halpern EJ, Mitchell DG, Wechsler RJ, Outwater EK, Moritz MJ, Wilson GA (2000) Preoperative evaluation of living renal donors: comparison of CT angiography and MR angiography. Radiology 216:434

17. Rankin SC, Jan W, Koffman CG (2001) Noninvasive imaging of living related kidney donors: evaluation with $\mathrm{CT}$ angiography and gadolinium-enhanced MR angiography. AJR Am J Roentgenol 177:349

18. Schlunt LB, Harper JD, Broome DR et al (2006) Multidetector computerized tomography angiography to predict lumbar venous anatomy before donor nephrectomy. J Urol 176:2576-2581

19. Dalrymple N, Prasad S, Freckleton MW et al (2005) Introduction to the language of three-dimensional imaging with multidetector CT. RadioGraphics 25:1409-1428

20. Fishman EK, Ney DR, Heath DG, Corl FM, Horton KM, Johnson PT (2006) Volume rendering versus maximum intensity projection in CT angiography: what works best, when, and why. Radiographics 26(3):905-922

21. Heath DG, Soyer PA, Kuszyk BS et al (1995) Three-dimensional spiral CT during arterial portography: comparison of three rendering techniques. RadioGraphics 15:1001-1011

22. Leclerc X, Godefroy O, Lucas C et al (1999) Internal carotid arterial stenosis: CT angiography with volume rendering. Radiology 210:673-682

23. Urban BA, Ratner LE, Fishman EK (2001) Three-dimensional volume-rendered $\mathrm{CT}$ angiography of the renal arteries and veins: normal anatomy, variants and clinical applications. RadioGraphics 21:373-386

24. Ofer A, Nitecki SS, Linn S et al (2003) Multidetector CT angiography of peripheral vascular disease: a prospective comparison with intraarterial digital subtraction angiography. AJR Am J Roentgenol 180:719-724

25. Johnson PT, Heath DG, Kuszyk BS, Fishman EK (1996) CT angiography with volume rendering: advantages and applications in splanchnic vascular imaging. Radiology 200:564-568

26. Kalender WA, Polacin A, Suss C (1994) A comparison of conventional and spiral CT: an experimental study on the detection of spherical lesions. J Comput Assist Tomogr 18:167-176, Published correction appears in J Comput Assist Tomogr 1994;18(4):671

27. Boiselle PM (2003) Multislice helical CT of the central airways. Radiol Clin N Am 41:561-574

28. Rubin GD (2003) 3-D imaging with MDCT. Eur J Radiol 45(suppl 1):S37-S41

29. Levoy M (1988) Display of surfaces from volume data. IEEE Comput Graph Appl 8:29-37

30. Cody DD (2002) AAPM/RSNA physics tutorial for residents: topics in CT. Image processing in CT. RadioGraphics 22:1255-1268

31. Soyer P, Heath D, Bluemke DA et al (1996) Three-dimensional helical CT of intrahepatic venous structures: comparison of three rendering techniques. J Comput Assist Tomogr 20:122-127

32. Felix W (1912) The development of the urogenital organs. In: Keibel F, Mall FP (eds) Manual of Human Embryology. J.B. Lippincott Company, Philadelphia, pp 752-880

33. Graves FT (1956) The anatomy of the intrarenal arteries in health and disease. Br J Surg 43:605-616

34. Boijsen E (1959) Angiographic studies of the anatomy of single and multiple renal arteries. Acta Radiol 181(suppl):1-135

35. Boijsen E (1983) Anomalies and malformations. In: Abrams HL (ed) Abrams angiography, 2nd edn. little, Brown, Boston, pp 1217-1221

36. Frick MP, Goldberg ME (1980) Uro- and angiographic findings in a "normal" population: screening of 151 symptom-free potential transplant donors for renal disease. AJR 134:503-505

37. Cuttino JT, Clark RL (1990) The normal vasculature of the genitourinary tract: embryology, anatomy, and hemodynamics. In: Hillman BJ (ed) Clinical urography, vol 3. Saunders, Philadelphia, pp 2076-2091

38. Raman SS, Pojchamarnwiputh S, Muangsomboon K, Schulam PG, Gritsch HA, Lu DS (2007) Surgically relevant normal and variant renal parenchymal and vascular anatomy in preoperative 16MDCT evaluation of potential laparoscopic renal donors. AJR Am J Roentgenol 188(1):105-114

39. Türkvatan A, Akinci S, Yildiz S, Olçer T, Cumhur T (2009) MDCT for preoperative evaluation of vascular anatomy in living renal donors. Surg Radiol Anat 31(4):227-235

40. Pollak R, Prusak BF, Mozes MF (1986) Anatomic abnormalities of cadaver kidneys procured for purposes of transplantation. Am Surg $52: 233-235$

41. Sebastià C, Peri L, Salvador R, Buñesch L, Revuelta I, Alcaraz A, Nicolau C (2010) Multidetector CT of living renal donors: lessons learned from surgeons. Radiographics 30:1875-1890

42. Kandpal H, Sharma R, Gamangatti S, Srivastava DN, Vashisht S (2008) Imaging the inferior vena cava: a road less traveled. Radiographics 28(3):669-689

43. El-Galley RES, Keane TE (2000) Embryology, anatomy, and surgical applications of the kidney and ureter. Surg Clin N Am 80:381-401

44. Kadir S (1986) Angiography of the kidneys. In: Kadir S (ed) Diagnostic angiography. Saunders, Philadelphia, pp 445-495

45. Kadir S (1991) Kidneys. In: Kadir S (ed) Atlas of normal and variant angiographic anatomy. Saunders, Philadelphia, pp 387-428

46. Dyer R (1993) Renal arteriography. In: Dyer R (ed) Basic vascular and interventional radiology. Churchill Livingstone, New York, pp 89-95

47. Sampaio FJ, Aragao AH (1990) Anatomical relationship between the renal venous arrangement and the kidney collecting system. J Urol 144:1089-1093 
48. Harrison LH, Flye MW, Seigler HF (1978) Incidence of anatomical variants in renal vasculature in the presence of normal renal function. Ann Surg 188:83-89

49. Abrams HL (1983) Renal venography. In: Abrams HL (ed) Abrams' angiography, 2nd edn. Little, Brown, Boston, pp $1327-$ 1364

50. Beckmann CF, Abrams HL (1979) Circumaortic venous ring: incidence and significance. AJR Am J Roentgenol 132:561565

51. Kahn PC (1973) Selective venography of the branches. In: Ferris EJ, Hipona FA, Kahn PC et al (eds) Venography of the inferior vena cava and its branches. Krieger, Huntington, pp 154-224
52. Minniti S, Visentini S, Procacci C (2002) Congenital anomalies of the venae cavae: embryological origin, imaging features and report of three new variants. Eur Radiol 12:2040-2055

53. Leventhal JR, Kocak B, Salvalaggio PR, Koffron AJ, Baker TB, Kaufman DB et al (2004) Laparoscopic donor nephrectomy 1997 to 2003: lessons learned with 500 cases at a single institution. Surgery $136: 881$

54. Bookstein JJ (1983) The roles of angiography in adrenal disease. In: Abrams HL (ed) Abrams angiography, 2nd edn. Little, Brown, Boston, pp 1395-1424

55. Notkovich $H$ (1956) Variations of the testicular and ovarian arteries in relation to the renal pedicle. Surg Gynecol Obstet 103:487-495 